\title{
A Resilience Model for Moderating Outcomes Related to Electronic Medical Record Downtime
}

\author{
Joseph M. WALSH ${ }^{\mathrm{a}, 1}$ and Elizabeth M. BORYCKI ${ }^{\mathrm{a}, \mathrm{b}}$ \\ a School of Health Information Science, University of Victoria, Victoria, BC, Canada \\ ${ }^{\mathrm{b}}$ Michael Smith Foundation for Health Research, Canada
}

\begin{abstract}
The objective of this scoping review is to develop a model to understand the factors that influence clinical downtimes or clinical activities in a healthcare organization. To report on the results of searches preformed using seven bibliographic databases, using the logical search criteria of (downtime AND (EMR OR Electronic Medical Record OR EHR OR Electronic Health Record). After a title, abstract and full-text review 26 articles remained. The articles were coded and analyzed for themes. Downtime planning activities mitigate the effects of disasters on patient safety outcomes and clinical delays. A model was developed representing the relationships between disasters, the moderating variable of downtime planning activities and patient safety as well as clinical outcomes. Disasters can have significant impact on patients and health professionals. Downtime planning activities can be enacted when a disaster occurs to moderate the effects of the downtime on patients and clinical activities and can improve safety.
\end{abstract}

Keywords. Downtime, safety, disasters, resilience

\section{Introduction}

A recent survey reported that, most North American healthcare institutions experienced an unplanned downtime in the past three years. Nearly $70 \%$ of those institutions who completed the survey stated they had experienced a downtime that lasted at least eight hours. Despite those figures, most organizations had not completely implemented a comprehensive downtime plan to address patient safety concerns during Electronic Medical Record (EMR) downtimes [1]. Downtimes can be caused by many different disasters from human error through to natural disasters such as flood, hurricane, and fire [2]. The Intergovernmental Panel on Climate Change (IPCC) refers to these types of environmental events as low-likelihood, high impact events and compound extreme events. Extreme weather events such as these are expected with high confidence to increase in frequency and amplitude according to the recent IPCC report on climate change [3]. During a downtime, some or all functions of an EMR can become unavailable. In both planned and unplanned downtime scenarios, clinical users risk timely access to critical patient information [4]. The objective of this scoping review is

\footnotetext{
${ }^{1}$ Corresponding author, Joseph M. Walsh, School of Health Information Science, University of Victoria, Victoria BC, Canada; E-mail: joewalsh@uvic.ca
} 
to develop a model to understand the factors that influence clinical downtimes or clinical activities in a healthcare organization.

\section{Methodology}

To understand the factors influencing clinical downtimes, we performed a scoping study following Arksey and O'Malley's 2005 methodology [5], including the optional consultation stage [6]. In this final stage, subject matter experts were consulted to further refine and interpret the key concepts discovered in the preceding stages. Following the final consultation stage with subject matter experts, a narrative review identified contextual factors and strategies that impact clinical operations during a clinical downtime. A model was created demonstrating the impact of downtime and how planning activities can be used to moderate the effects of a downtime.

\section{Results}

To capture a broad set of results, searches were preformed using seven different bibliographic databases, using the logical search criteria of (downtime AND (EMR OR Electronic Medical Record OR EHR OR Electronic Health Record)). 672 articles were returned from all sources, with 625 remaining after duplicates were removed.

The remaining results were screened by title, abstract and full text for context. Articles selected must be published in a peer reviewed journal, printed in English, and have abstracts and full text available electronically. One reviewer performed all screening stages, with 30 articles remaining after title and abstract screening. Four additional articles were removed following a full text review as these articles did not discuss technology downtimes in the context of hospital or clinical operations. The remaining 26 articles, published between January 2008 and March 2021, were coded and analyzed for themes.

\section{Discussion}

The literature shows several patient safety outcomes are affected by downtimes. The theme of patient safety was common in the publications reviewed and included the subthemes of clinical error, patient harm, clinical delays, and data integrity. One study concluded that "downtime-exposed patients had statistically significant longer intraoperative courses and postoperative hospital length of stay" [7]. Many of the included articles directly discuss patient safety during downtime scenarios [8]. Errors occurring during a clinical downtime affect a clinician's ability to access the most relevant patient information. Wang et al. identified "recent surveys of US hospitals have linked downtime to medication errors, increased length of stay and patient harm." [9].

There are several ways that clinical delays affect patient care. Delays can occur in the reporting of test results, length of stay, diagnosis, and treatment. Larsen et al. state that "patients in emergency situations, whose diagnosis depends on timely laboratory results are exposed to significant risks" [10]. Researchers identified the importance of downtime planning such as mitigation in reducing the effects of a downtime on clinical 
activities. Mitigation of clinical downtime is considered any action taken before, during, or after a downtime to minimize the impact or duration of a downtime event [11]. Downtime planning is a mitigation strategy commonly discussed in the literature. These downtime plans should be reviewed regularly so that the plans remain current to the evolving clinical workflows and applications [12]. Downtime kits including relevant policy, procedure, and preprinted forms required during a downtime should also be checked and updated regularly. Downtime planning can also include proactive steps taken to build out systems redundancy with backup or clustered systems. One common backup system is the use of uninterruptable power supplies and standby emergency power generators. Other backup systems are online and offline data repositories to use as alternate sources for patient data when the primary system is offline, or for data recovery after data loss or corruption $[4,13]$. Clustered servers can also be deployed so that routine maintenance can be performed without affecting application availability. Standby clustered or redundant systems can be staged at the same site, or for greater fault tolerance, at a secondary data center. In some cases, it can take time to bring these standby systems fully online and accessible by clinical users $[1,4,14]$. Routine data backups can be performed to disk, or on removable media such as tape so that it can be taken offline to preserve the historical data integrity for recovery at a later time.

In summary downtimes increase surgical times and postoperative length of stay [7], adverse clinical outcomes, clinical errors [15], medication errors [4] and adverse patient outcomes [9]. We also found that downtime can contribute to delays in patient test results [4], diagnosis [10] and treatment [9]. The scoping review study, revealed that several moderating variables were found to influence the outcomes of a disaster on patients and health professionals. They include contingency planning [16], mitigation [17], systems redundancy [14] and backup systems [1]. Their interactions are shown in figure 1 in the model we created after reviewing the literature. Disasters lead to a number of patient safety outcomes, including adverse patient outcomes such as increased surgical time and length of stay as well as clinical errors such as medication errors and adverse patient outcomes. It was also discovered that downtimes led to delays in patient test results, diagnosis and treatment (see Figure 1). Of note, researchers found that downtime planning (i.e. contingency planning, mitigation, systems redundancy and backup systems) moderates the effect of a downtime on patient safety and clinical errors.

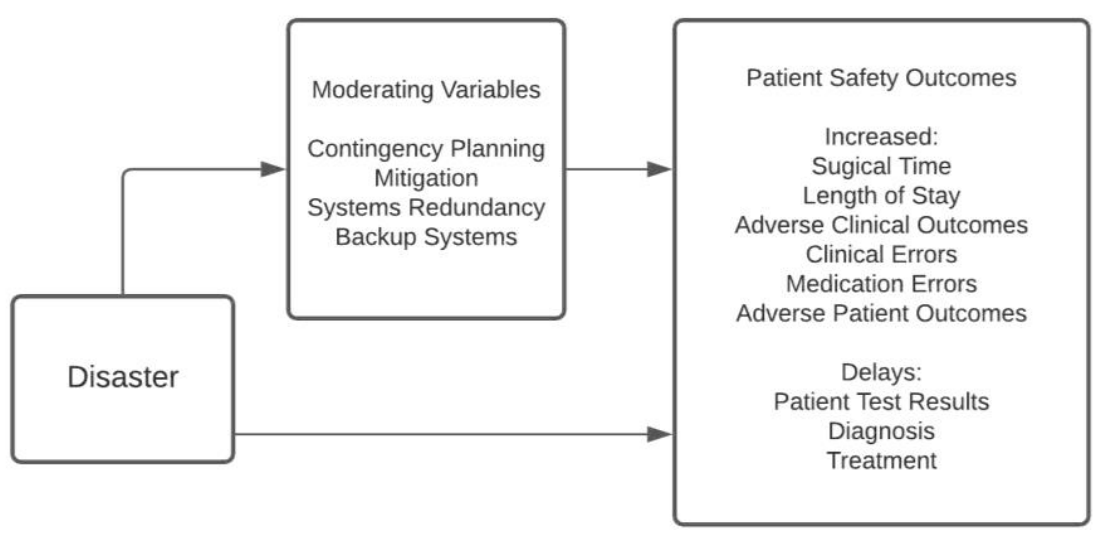

Figure 1. Disaster's effect on patient safety outcomes with moderating variables 


\section{Conclusion}

Downtime planning activities such as contingency planning, mitigation, systems redundancy, and backup systems moderate the effects of a disaster on patient safety outcomes and delays in critical patient care activities. In this scoping review we identified the key findings (or themes) in the literature that need consideration when developing downtime plans and integrating them into healthcare organizational structure. Of note, we created a model that shows the impacts of a downtime and how downtime planning activities can moderate the effects of a downtime on patient safety outcomes and health professional clinical activities. More work needs to be done in the area of studying the effects of downtime planning and preparation and its effect on patient safety outcomes. Future research for our group includes fully testing the models derived from the literature as well as identifying new ways of moderating the effects of downtimes.

\section{Acknowledgements}

The authors acknowledge NSERC funding, reference number RGPIN-2020-06950. E. Borycki receives funding from the Michael Smith Foundation for Health Research.

\section{References}

[1] Sittig DF, Gonzalez D, Singh H. Contingency planning for electronic health record-based care continuity: a survey of recommended practices. International Journal Of Medical Informatics. 2014;83(11):797-804.

[2] Cleary PA. Lessons learned from unscheduled downtime -- Data inaccessible for up to 10 days. Hospital Access Management. 2016;35(4):40-1.

[3] IPCC. The Physical Science Basis. Contribution of Working Group I to the Sixth Assessment Report of the Intergovernmental Panel on Climate Change. Press.: IPCC; 2021.

[4] Larsen E, Fong A, Wernz C, Ratwani RM. Implications of electronic health record downtime: an analysis of patient safety event reports. Journal Of The American Medical Informatics Association: JAMIA. 2017;25(2):187-91.

[5] Arksey H, O'Malley L. Scoping studies: towards a methodological framework. International Journal of Social Research Methodology. 2005;8(1):19-32.

[6] Levac D, Colquhoun H, O'Brien KK. Scoping studies: advancing the methodology. Implement Sci. 2010;5(1):69.

[7] Harrison AM, Siwani R, Pickering BW, Herasevich V. Clinical impact of intraoperative electronic health record downtime on surgical patients. Journal Of The American Medical Informatics Association: JAMIA. 2019;26(10):928-33.

[8] Scantlebury A, Sheard L, Fedell C, Wright J. What are the implications for patient safety and experience of a major healthcare IT breakdown? A qualitative study. Digital Health. 2021;7:20552076211010033.

[9] Wang Y, Coiera E, Gallego B, Concha OP, Ong M-S, Tsafnat G, et al. Measuring the effects of computer downtime on hospital pathology processes. Journal Of Biomedical Informatics. 2016;59:308-15.

[10] Larsen E, Haubitz C, Wernz C, Ratwani R, editors. Improving Electronic Health Record Downtime Contingency Plans with Discrete-Event Simulation. 49th Annual Hawaii International Conference on System Science; 2016 2016; Hawaii: IEEE.

[11] Walsh JM, Borycki EM, Kushniruk AW. Effects of Electronic Medical Record Downtime on Patient Safety, Downtime Mitigation, and Downtime Plans. International Journal of Extreme Automation and Connectivity in Healthcare. 2020;2(1):161-86.

[12] Fernández MT, Gómez AR, Santojanni AM, Cancio AH, Luna DR, Benítez SE. Electronic Health Record System Contingency Plan Coordination: A Strategy for Continuity of Care Considering Users' Needs. Studies in health technology and informatics. 2015;216:472-6.

[13] Campos F, Luna D, Sittig DF, Bernaldo de Quirós FG. Design, Implementation and Evaluation of an Architecture based on the CDA R2 Document Repository to Provide Support to the Contingency Plan. Studies In Health Technology And Informatics. 2015;216:173-7. 
[14] Coffey PS, Postal S, Houston SM, McKeeby JW. Lessons Learned from an Electronic Health Record Downtime. Perspectives in Health Information Management. 2016:1-7.

[15] Fahrenholz CG, Smith LJ, Tucker K, Warner D. Plan B. A practical approach to downtime planning in medical practices. J AHIMA. 2009;80(11):34-8.

[16] Dave K, Boorman RJ, Walker RM. Management of a critical downtime event involving integrated electronic health record. Collegian. 2020;27(5):542-52.

[17] Walsh JM, Borycki EM, Kushniruk AW. Strategies in Electronic Medical Record Downtime Planning: A Scoping Study. Studies In Health Technology And Informatics. 2019;257:449-54. 